Journal of

Case Reports: Open Access

\title{
Primary Leiomyosarcoma of the Left Atrium
}

\section{Crizel Denise Uy*, Fernando Gracieux Y}

Section of Medical Oncology, Department of Medicine, University of the Philippines Manila, Philippine General Hospital

${ }^{*}$ Corresponding author: Crizel Denise Uy, University of the Philippines - Philippine General Hospital, philippines, Tel: 09954140946, E-mail: crizeldenise@gmail.com

Received Date: September 12, 2019 Accepted Date: October 26, 2019 Published Date: October 28, 2019

Citation: Uy Crizel Denise V (2019) Primary Leiomyosarcoma of the Left Atrium. Case Reports: Open Access 4: 1-4.

\begin{abstract}
Objectives: To present a case of a leiomyosarcoma of the left atrium, presenting as exertional dyspnea in a middle-aged Filipino. This study likewise presents a review of current literature.

Results: A 44 years old female presented with a three-day history of exertional dyspnea. Transesophageal echocardiography revealed a mass with pericardial effusion and a large mass occupying almost the entire left atrial cavity. She underwent complete excision with mitral valve repair. Histopathology revealed bundles of fascicles of spindle-shaped cells with eosinophilic infiltrates, positive for SMA, Caldesmon, and Vimentin, and a high Ki67 of >50\% -- consistent with primary leiomyosarcoma. No adjuvant chemotherapy was done. Within three months of post-operation and lost to follow-up, the patient noted the recurrence of dyspnea. Echocardiography revealed the recurrence of the mass, again obstructing the left atrium. Metastatic lesions were also on both lungs. The patient was planned a 2nd resection of the mass, followed by systemic chemotherapy, but died due to obstructive heart failure.
\end{abstract}

Summary/conclusion: Primary cardiac leiomyosarcomas (PCLMS) are extremely rare tumors. Studies reveal female preponderance with mean age of 48 years. The tumors seem to originate from the cardiac chambers, without the involvement of the pericardium and great vessels. Increased tumor burden, metastatic disease, high proliferative index, and high-grade tumors usually translate to a worse prognosis. Complete surgical resection of the tumor is still the mainstay of treatment followed by doxorubicin, ifosfamide, or dacarbazine-based regimens.

C2019 The Authors. Published by the JScholar under the terms of the Creative Commons Attribution License http://creativecommons.org/licenses/ by $/ 3.0 /$, which permits unrestricted use, provided the original author and source are credited. 


\section{Introduction}

Tumors involving the heart are rare occurring entities. Cardiac masses are 40-100 times more frequently caused by metastases from a primary site --- usually either the breast, lung, or kidneys [1]. Primary cardiac tumors have an estimated frequency of only $0.0017-0.33 \%$, of which $75 \%$ are benign [2]. Among the small percentage of primary malignant tumors, the most frequently occurring histologic subtypes include rhabdomyosarcomas, mesotheliomas, and fibromyosarcomas [3]. Primary leiomyosarcomas of the heart occur in less than $1 \%$ of the malignant cases, usually arising from the left atrial chamber [1-3]. While a leiomyosarcoma is a common form of mesenchymal tumor arising from smooth muscle, there is a paucity of literature in defining outcomes of non-uterine involvement [4]. Leiomyosarcomas of the heart usually present at an advanced stage resulting in dismal prognoses [4].

Here we report a case of primary leiomyosarcoma of the left atrium in a 44 year old female presenting as acute onset exertional dyspnea.

\section{Case report}

A 44-year-old female presented with a 3-day history of progressive dyspnea noted upon exertion. Increased severity of dyspnea prompted medical consult, and an echocardiography revealed a massive pericardial and bilateral pleural effusions. Transthoracic echocardiography likewise showed a very large lobulated mass occupying most of the left atrium, extending into the mouth of the left atrial annulus, pushing the inter-atrial septum towards the right atrium. The patient underwent an emergency pericardiocentesis, followed by surgical excision of the mass and mitral valve repair. Grossly, the intra-cardiac mass measured $4 \times 2 \times 2 \mathrm{~cm}$ and weighed 15 grams. Sections revealed bundles of fascicles of spindle-shaped cells with eosinophilic cytoplasm, pleomorphic nuclei, and frequent mitotic figures. Giant cells with areas of necrosis were likewise seen. Immunohistochemistries revealed positive for SMA, Caldesmon, and Vimentin; while negative for myoglobin, myogenin, desmin, CD 34, Myo D1, and S100 - compatible with a primary cardiac leiomyosarcoma. Ki67 was high at $>50 \%$. The patient had episodes of atrial fibrillations post-operatively and was treated with anti-coagulants. A complete workup was done, and no signs of metastatic disease were present.

She sought to consult at our clinic two months postop, seeking oncologic consult. She was asymptomatic with good functional status. Cardiac examination revealed a normal heart rate, regular rhythm, with distinct heart sounds. S1 > s2 at the apex, s2>s1 at the base, with no murmurs appreciated. Echocar- diography revealed a recurrent left atrial mass measuring $1.86 \mathrm{x}$ $1.80 \mathrm{~cm}$, while the rest of the structures were unremarkable. A metastatic workup was likewise negative for disseminated disease. The patient was not amenable to undergo re-excision of the mass nor receive chemotherapy as advised.

Three months post-op the patient complained of recurrent dyspnea, orthopnea, and easy fatigability. Repeat echocardiography revealed an increase in the size of the left atrial mass, this time measuring $3.9 \times 3.6 \mathrm{~cm}$ at widest diameter. Metastatic workup likewise revealed disseminated disease to bilateral lungs and liver. Due to cardiac outlet obstruction caused by the progressive tumor burden, the patient succumbed to death.

\section{Discussion}

Cardiac masses are rare tumors, with a low frequency of only $0.0017-0.33 \%$ of the population. The majority of these tumors account for metastatic lesions from a known primary site, usually from the breast, lung, or kidneys. Primary cardiac lesions are also usually benign, as primary malignant tumors account for only 25\% [1-4]. A leiomyosarcoma is an aggressive form of soft tissue sarcoma derived from smooth muscle cell and of mesenchymal origin. They usually arise from the uterus, gastrointestinal tract, and retroperitoneum, and very rarely do they originate from the heart [5]. Primary cardiac leiomyosarcomas are exceedingly rare entities with an incidence of only $0.025 \%$ of all cardiac sarcomas, with only a bleak number of reported cases [6].

A systematic review conducted by Wang et al in 2015 among 79 reported cases, revealed a median age of 48 years upon presentation, with a slight female predominance [4]. Initial presenting symptoms are usually brought about by the obstructive nature of the lesion, resulting in dyspnea, cough, chest pain, and edema. Arrhythmia-related symptoms such as palpitations and tachycardia were also present in a minority of patients (12.5\%). Other non-specific symptoms included fatigue, malaise, fever, and sweating $[4,7,8]$ Although primary leiomyosarcomas most commonly arise from the left atrium in around 59\% of reported cases, they may also arise from the right ventricle (20.2\%), right atrium (15.2\%), and left ventricle (5.1\%) [4, 9] Leiomyosarcomas are characterized by slow, insidious growth. And due to its oligosymptomatic course, these tumors are rarely diagnosed early and usually present at an advanced stage, or confirmed only during an autopsy [9].

Transthoracic two-dimensional echocardiography is currently the most useful examination in the diagnosis of cardiac 


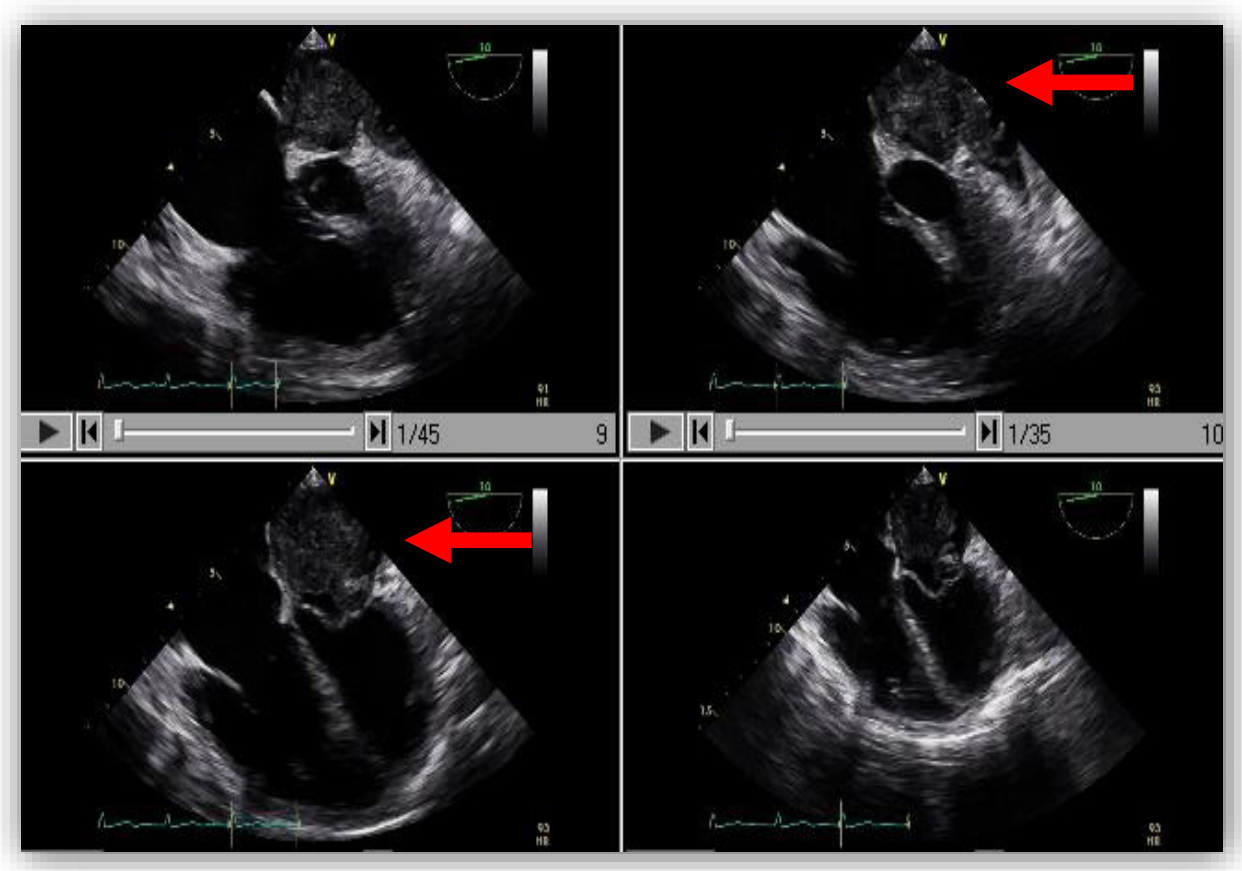

Figure 1. Transesophageal echocardiogram showed a very large lobulated mass occupying most of the left atrium and also extending into the mouth of the left atrial annulus, pushing the inter-atrial septum towards the RA. The mass seems to originate by a wide base from the lower part of the IAS and MV annulus.

tumors, offering a high sensitivity of almost $100 \%$. It can determine the tumor's location, size, shape, movement, and attachment tocardiac structures [10-12]. While the value of transthoracic two-dimensional echocardiography (TTE) is well established in cardiac tumors, the transesophageal approach may offer optimal visualization of the tumor in some cases. The unobstructed view from transesophageal echocardiography allows excellent visualization of structures not well seen on TTE, with a higher resolution [13]. CT scan and cardiac MRI may also provide further information about morphology, location, and extent of the mass [16].

Macroscopic characteristics of cardiac leiomyosarcomas reveal a wide-based tumor, usually originating from the pulmonary veins. Histopathologic characteristics are consistent with tumors of mesenchymal origin, in which malignant cells may appear spindle, epitheloid, or pleomorphic [1,4]. Immunohistochemistries offer valuable information in diagnosing cardiac leiomyosarcomas, with SMA, MSA, and Calponin being the most sensitive markers [14-15]. Other useful, albeit less sensitive markers for sarcomas, include vimentin, caldesmon, myosin, S100 proteins, CD68, CD31, CD34, CD68, and EMA [14-15].

Due to the rarity of primary cardiac leiomyosarcomas, there have been no large cohort studies to date [4]. A multi-disciplinary approach with thoracic cardiovascular surgery, medical oncology, and radiation oncology are optimal. Complete surgical resection, whenever possible, is still the mainstay of treatment. Leiomyosarcomas belong to a high-risk group of soft tissue sarcomas with chemosensitive histology. Cytotoxic agents recommended include Doxorubicin, Ifosfamide, and Dacarbazine [16-17]. Indications for radiotherapy are controversial, and are mostly reserved for the palliative setting [16]. In reported cases, the mean duration of survival is only 12 months after optimal surgery and chemotherapy. Tumor related factors such as high tumor grade, increased mitotic index, poor surgical margins, and presence of distant metastases, all account for worsened prognosis [15-17].

\section{Conclusion}

Primary cardiac leiomyosarcomas are extremely rare entities, with only a small number of reported cases. These lesions most commonly present at advanced stages with symptoms compatible with cardiac obstruction. The mainstay of treatment is still a complete surgical resection, followed by chemotherapy. Despite multimodality treatment, however, prognoses remain dismal with an average survival of 1 year from the time of diagnosis. 


\section{References}

1. Braun R, Rivera C, Frausto A, Rodriguez A, et al. (2017) Immunohistochemical diagnosis of primary cardiac leiomyosarcoma in a Latin America patient. Rare Tumors Volume 9: 6669.

2. Reynen K (1996) Frequency of primary tumors of the heart. Am J Cardiol 77:107.

3. Andersen R, Kristensen B, Gill S (2013) Cardiac leiomyosarcoma - a case report. Int J Clin Exp Pathol 6: 1197-1199.

4. Wang J, Cui L, Jiang T, Li Y, Wei Z (2015) Primary cardiac leiomyosarcoma: An analysis of clinical characteristics and outcome patterns. Asian Cardiovascular and Thoracic Annals 23: 623-630.

5. Gustafson P, Willen H, Baldetrop B, et al. (1992) Soft tissue leiomyosarcoma: a population-based epidemiologic and prognostic study of 48 patients, including cellular DNA content. Cancer 70:114.

6. Zaidi S, Husain S, Barakah D (2017) Primary leiomyosarcoma of the left atrium with heterologous differentiation. Annals Saudi Med 37: 403-405.

7. Hudzik B, Miszalski-Jamka K, Glowacki J, et al. (2015) Malignant tumors of the heart. Cancer Epidemiol 39: 665-672.

8. Bossert T, Gummert JF, Battellini R, et al. (2005) Surgical experience with 77 primary cardiac tumors. Interact Cardiovasc Thorac Surg 4: 311-315.

9. Gierlak W, Suminska J, Zielinski P, et al (2015) Cardiac tumors: leiomyosarcoma - a case report. Kardiochirurgia i Torakochirurgia Polska 12: 251-254.

10. Attar MN, Sharman DC, et al. (2007) A rare case of multiple right heart myxomas. International Journal of Cardiology e66-67.

11. Kiyan S and Aksay E (2007) Left atrial myxoma. The journal of Emergency Medicine

33: 73-74.

12. Whitlock R, Evans R, et al. (2007) Giant left atrial myxoma and associated mitral valve pathology. Journal of Cardio- thoracic and Vascular Anesthesia 21: 103-105.

13. DeVille J, Corely D, Jin B, et al (1995) Assessment of Intracardiac masses by trans esophageal echocardiography. Tex Heart Inst J 22: 134-137.

14. Carvalho JC, Thomas DG, Lucas DR (2009) Cluster analysis of immunohistochemical markers in leiomyosarcoma delineates specific anatomic and gender subgroups.Cancer 115: 4186-4195.

15. Donsbeck AV, Ranchere D, Coindre JM, et al. (1999) Primary cardiac sarcomas: an immunohistochemical and grading study with long-term follow-up of 24 cases. Histopathology 34: 295-304.

16. Behi K, Ayadi M, Menzi E, et al. (2017) Two years of survival of primary cardiac leiomyosarcoma managed by surgical and adjuvant therapy. Clinical Sarcoma Research 7:5.

17. Steen S, Stephenson G (2008) Current treatment of soft tissue sarcoma. Proc Bayl Univ Med Cent. 21: 392-396.

18. Lv Y, Pang X, Zhang Q, Jia D (2015) Cardial leiomyosarcoma with multiple lesions involved: a case report. Int J Clin Exp Pathol 8: 15412-15416.

\footnotetext{
Submit your manuscript to a JScholar journal and benefit from:

ฯ Convenient online submission

ब Rigorous peer review

I Immediate publication on acceptance

- Open access: articles freely available online

- High visibility within the field

ब Better discount for your subsequent articles

Submit your manuscript at http://www.jscholaronline.org/submit-manuscript.php
} 